\title{
Spectral Flow and Index Theorem for Staggered
} Fermions

\section{E. Follana*}

Universidad de Zaragoza

E-mail: efollana@unizar.es

\section{Azcoiti}

Universidad de Zaragoza

E-mail: azcoitidazcoiti.unizar.es

\section{G. Di Carlo}

INFN, Laboratori Nazionali del Gran Sasso

E-mail: giuseppe.dicarlo@lngs.infn.it

\section{A. Vaquero ${ }^{\dagger}$}

Universidad de Zaragoza

E-mail: alexveunizar.es

We investigate numerically the spectral flow introduced by Adams for the staggered Dirac operator on realistic gauge configurations. We study both the unimproved and the HISQ Dirac operators. We compare the spectral flow index with the index obtained by identifying low-lying modes of large chirality.

XXIX International Symposium on Lattice Field Theory

July 10-16 2011

Squaw Valley, Lake Tahoe, California

\footnotetext{
*Speaker.

$\dagger$ Present address: The Cyprus Institute.
} 


\section{Introduction}

In [1], Adams introduced a new definition of topological charge for lattice gauge fields based on the spectral flow of a hermitian operator related to the staggered Dirac operator. Some numerical results were obtained there for synthetic configurations in the $2 D \mathrm{U}(1)$ model.

Here we present preliminary ${ }^{1}$ numerical results in realistic, $4 D$ pure gauge $S U(3)$ configurations, confirming the good properties of Adams' definition, and the agreement of the index calculated with the new definition and by counting the number of low-lying modes of high chirality.

\section{Definition of the topological charge}

The hermitian operator introduced in [1] is defined by

$$
H_{s t}(m)=i D_{s t}-m \Gamma_{5}
$$

where $D$ is the massless staggered Dirac operator and $\Gamma_{5}$ is the taste-singlet staggered $\gamma_{5}[3]$. This operator is hermitian, and we can study its spectral flow, $\lambda(m)$. The would-be zero modes of $D_{s t}$ are now identified with the eigenmodes for which the corresponding eigenvalue flow $\lambda(m)$ crosses zero at low values of $m$, and the chirality of any such mode equals (with our conventions) the sign of the slope of the crossing [1].

For the most part we work with the highly improved Dirac operator (HISQ) [4], although for comparison we will also show some results corresponding to the unimproved (1-link) Dirac operator.

To compare with previous work, we also calculate the low-lying modes of the HISQ Dirac operator at $m=0$, and identify the would-be zero modes with the high taste-singlet chirality ones $[5,6]$.

\section{Results}

For our numerical calculations we use configurations from an ensemble of tree-level Symanzik and tadpole improved quenched QCD with a lattice spacing of approximately $0.077 \mathrm{fm}$ [5].

The operator $H_{s t}(m)$ is hermitian, and its low-lying eigenmodes are easily calculated numerically with standard methods. Its spectrum has the exact symmetry $\lambda(m) \leftrightarrow-\lambda(-m)$, therefore we only show results for $m<0$. An equal number of crossings, with identical slope, will be present for $m>0$.

We show in figures 1, 2 and 3 the results obtained for three configurations corresponding (a posteriori), to topological charge $0,-1$, and 2 . We can clearly see the agreement between both definitions of the topological charge, with the expected $4 Q$ high-chirality modes, and $4 Q$ crossings at low $m$.

In order for the topological charge to be well defined by the spectral flow, it is necessary that the crossings at low values of the mass and other possible crossings at larger values of the mass are well separated. We show in figure 4 the spectral flow for the same gauge configuration

\footnotetext{
${ }^{1}$ More complete results will be presented in [2].
} 

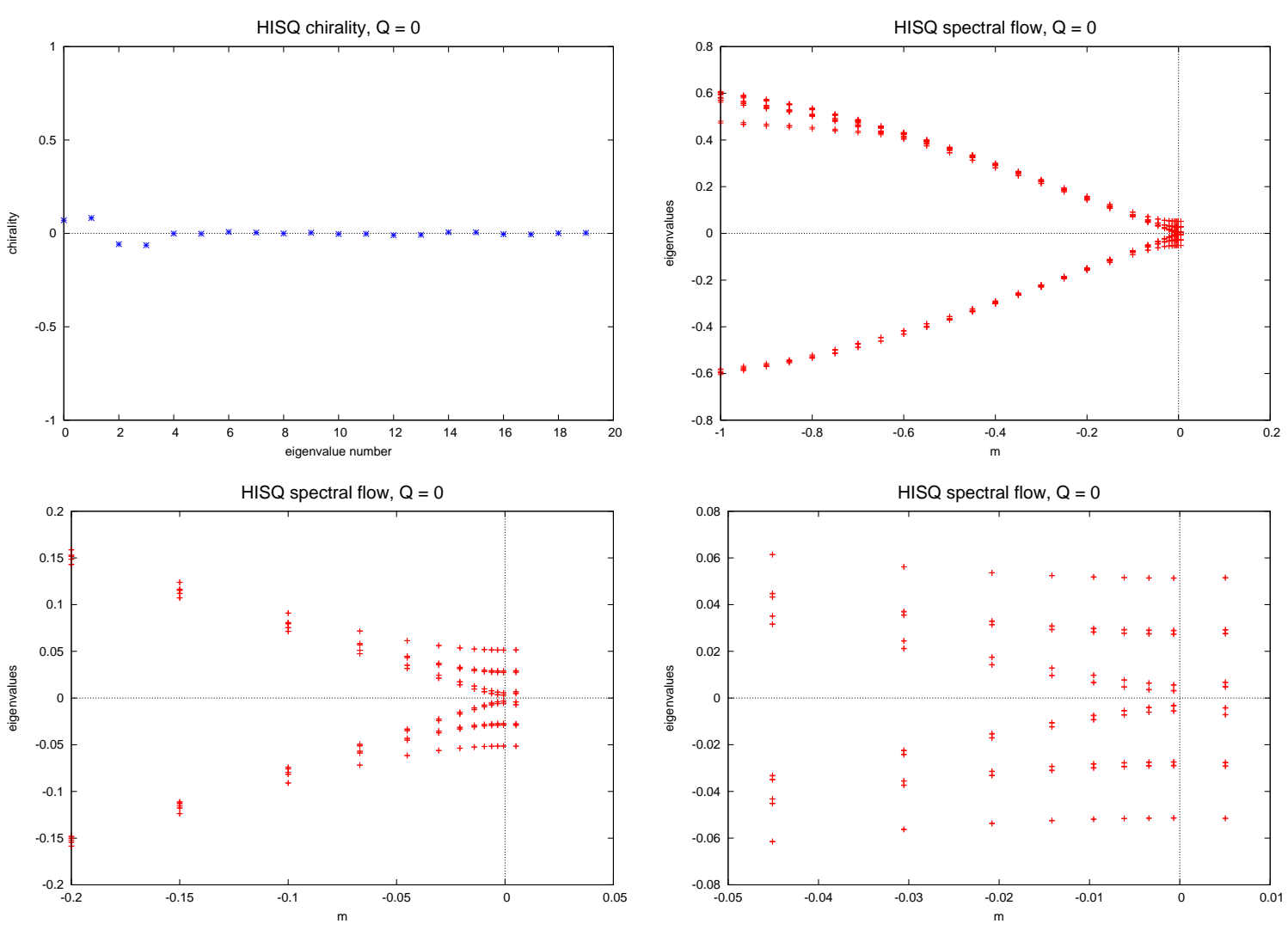

Figure 1: Top left figure: taste-singlet chirality for the low-lying modes of the HISQ Dirac operator (only half of the modes are shown, as the other half is exactly degenerate, due to an exact symmetry of the Dirac action.) Top right and bottom figures: spectral flow for the low-lying modes of the corresponding hermitian operator $H_{s t}(m)$, for various ranges of $m$ (we only show the range $m<0$, due to the exact symmetry $\lambda(m) \leftrightarrow-\lambda(-m))$. This is for a gauge configuration with $Q=0$.

corresponding to $Q=-1$, but with a much larger mass range. We see that there is no sign of any other crossing until a very large value of $m$, of order $\mathscr{O}(200)$. We conclude that, at least at this lattice spacing and for the HISQ Dirac operator, there is a very good separation between low and high mass crossings.

In figure 5 we compare the spectral flow coming from the HISQ and the 1-link Dirac operators, on the same gauge field configuration, of topological charge -1. Both flows agree on the value of the topological charge of the configuration, but the crossings corresponding to HISQ take place at a much smaller value of $m$. This is according to expectations, because in the continuum limit the only possible crossing is at $m=0$, and we expect the HISQ operator to be much closer to the continuum than the 1-link operator. Another manifestation of this is the fact that the four-fold degeneracy of the continuum theory is also much more closely approximated by the HISQ action, due to its much reduced taste-symmetry breaking.

The definition of topological charge through the identification of the high-chirality, low-lying modes of the Dirac operator works well in practice, and any ambiguities are expected to vanish in 

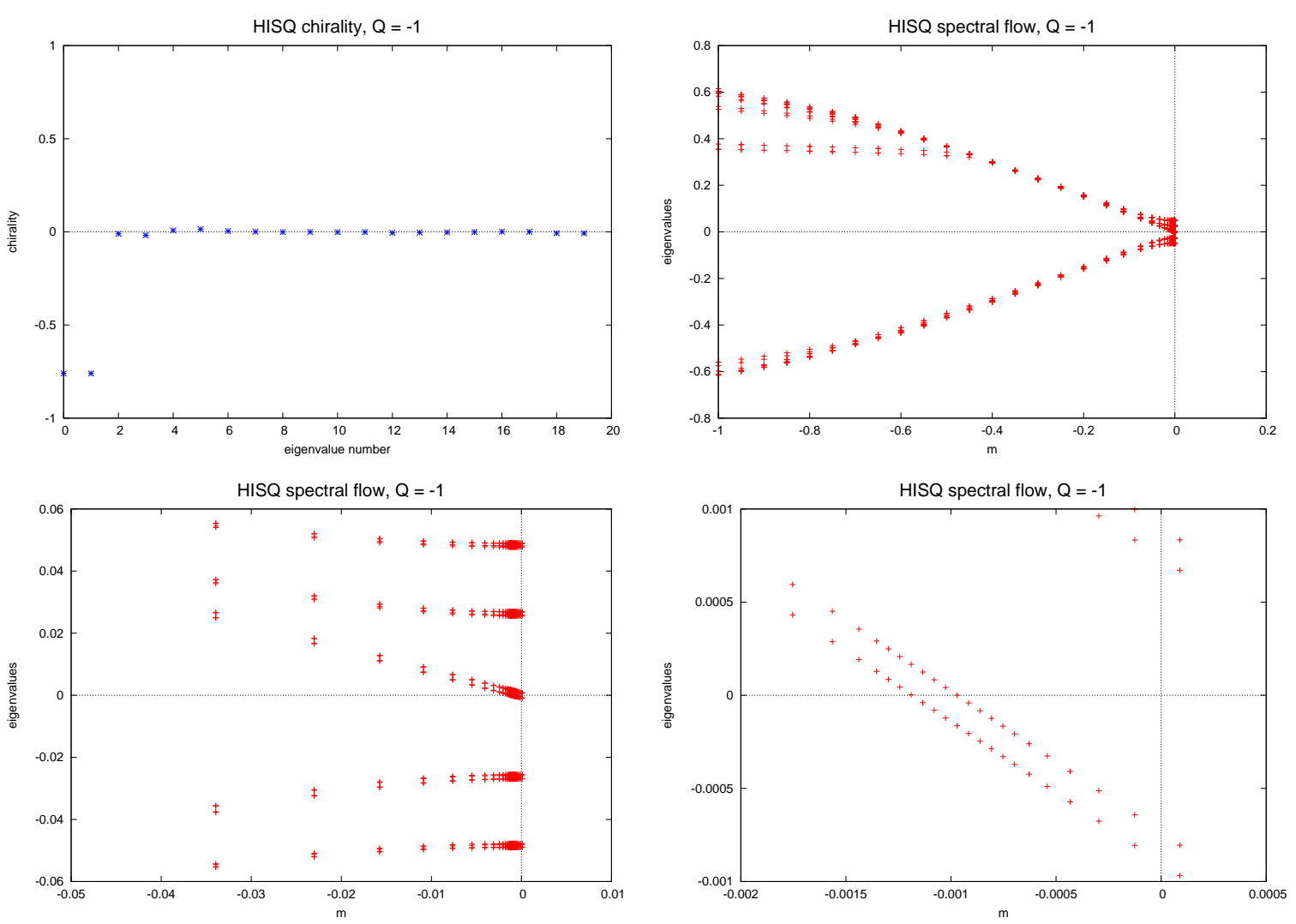

Figure 2: Top left figure: taste-singlet chirality for the low-lying modes of the HISQ Dirac operator. Top right and bottom figures: spectral flow for the low-lying modes of the corresponding hermitian operator $H_{s t}(m)$, for various ranges of $m$. This is for a gauge configuration with $Q=-1$.

the continuum limit as $a^{2}$, where $a$ denotes the lattice spacing. Nevertheless, at finite lattice spacing there are a few configurations for which the classification in a topological sector is not clear-cut [5]. In figure 6 we show the chiralities and spectral flow for one of those configurations. The high-chirality criterion would indeed be ambiguous applied to this configuration. The spectral flow criterion is always well-defined ${ }^{2}$, and would assign a topological charge 0 to this configuration. We can see, however, that this is the result of having pairs of crossings with opposite slopes, instead of not having any crossing (as is the case in figure 1 for the configuration with $Q=0$ ).

\section{Conclusions and Outlook}

We have presented preliminary numerical evidence that Adams' definition of the topological charge using the staggered Dirac operator works as expected for realistic (quenched) $S U$ (3) gauge fields. The crossings corresponding to low and high-lying modes are well separated, and therefore the topological charge of a configuration is unambiguously defined, even in cases which are ambiguous using other definitions.

\footnotetext{
${ }^{2}$ At least as long as there is a clear-cut separation between low-modes and high-modes.
} 

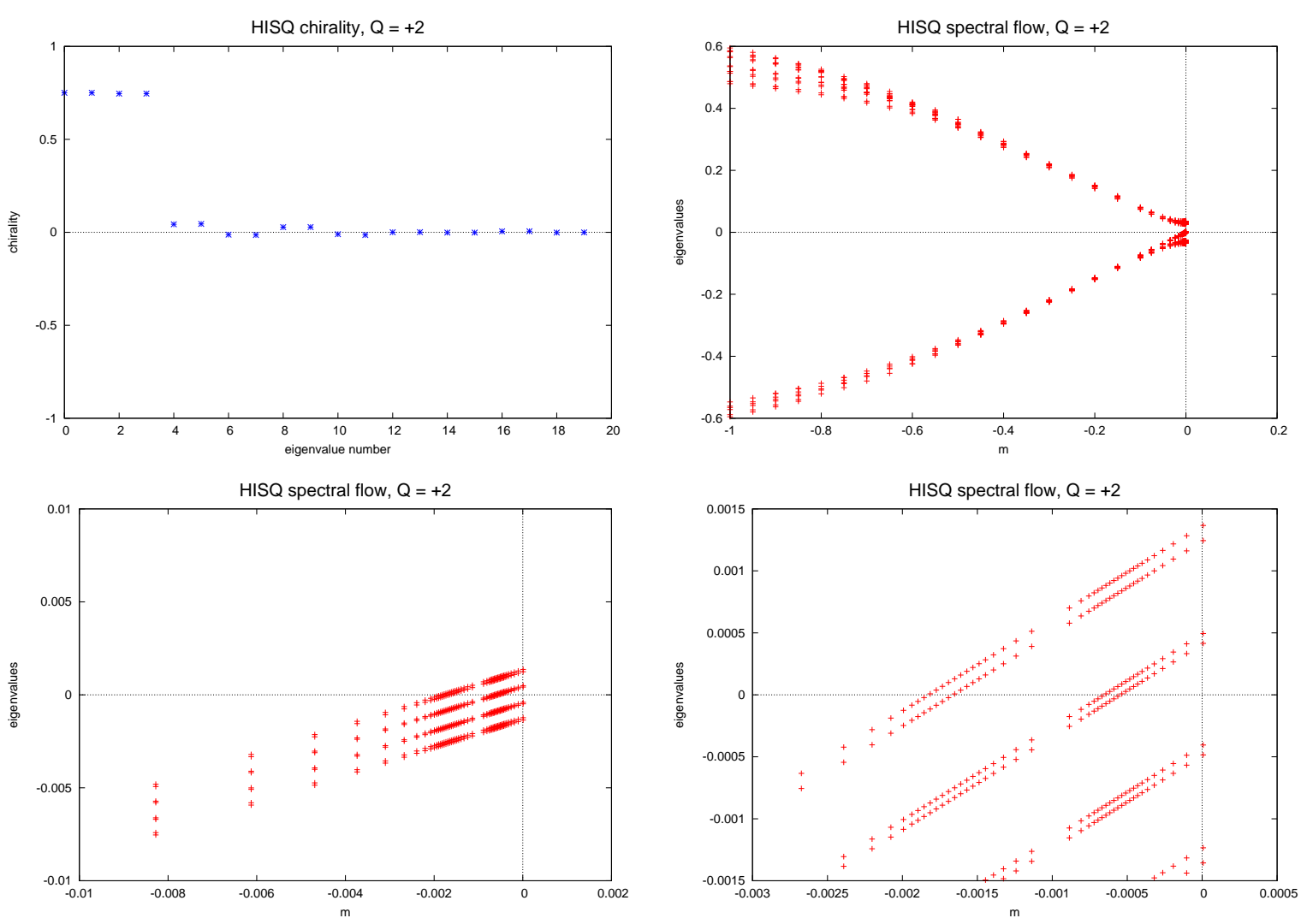

Figure 3: Top left figure: taste-singlet chirality for the low-lying modes of the HISQ Dirac operator. Top right and bottom figures: spectral flow for the low-lying modes of the corresponding hermitian operator $H_{s t}(m)$, for various ranges of $m$. This is for a gauge configuration with $Q=+2$.

It would be interesting to compare the staggered Dirac spectral flow with the usual Wilson Dirac spectral flow on the same gauge configurations, as well as studying the dependence on the lattice spacing [2].

Inspired by this definition of the spectral flow, one can define an overlap operator starting with a staggered kernel, instead of the usual Wilson one [7], producing a chiral operator representing two tastes of fermions. A similar construction can be carried out to further reduce the degeneracy and produce a one-flavour overlap operator [8]. The question now is whether this construction is numerically advantageous as compared with the usual overlap construction. Preliminary results are presented in [9].

\section{Acknowledgments}

We thanks Alistair Hart for generating the configurations. This work was funded by an INFNMICINN collaboration, MICINN (under grants FPA2009-09638 and FPA2008-10732), DGIIDDGA (grant 2007-E24/2), and by the EU under ITN-STRONGnet (PITN-GA-2009-238353). E. 


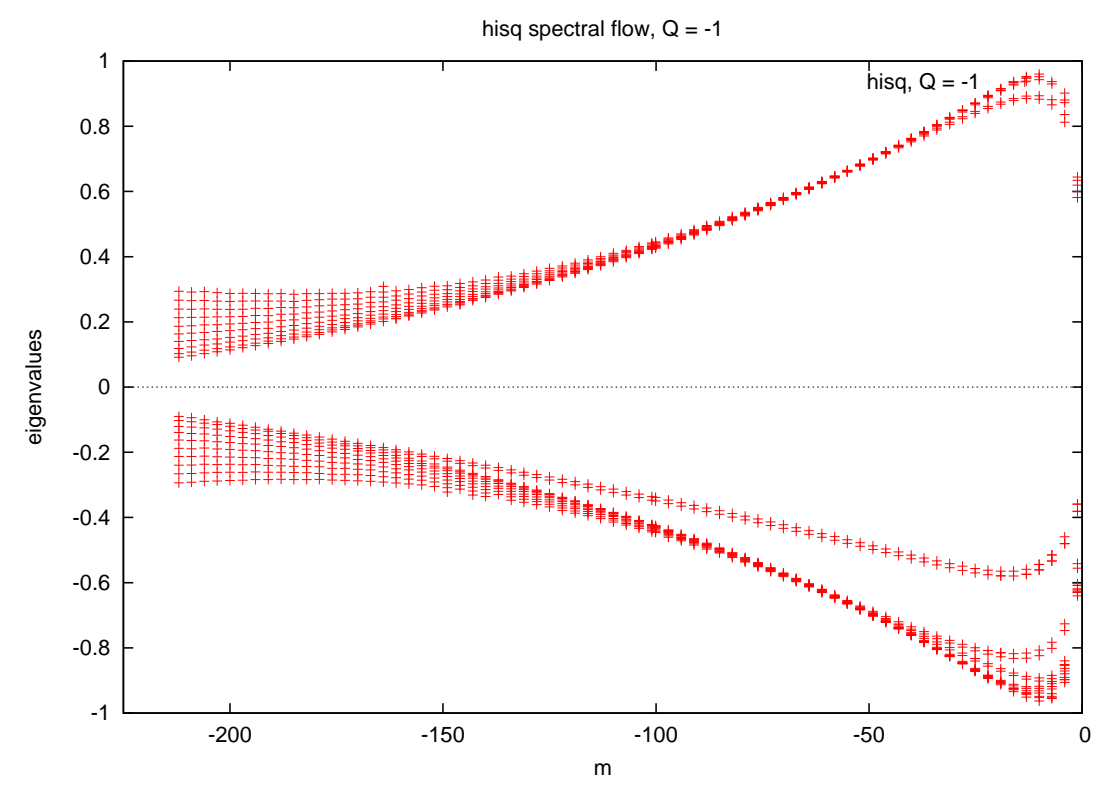

Figure 4: Spectral flow corresponding to the HISQ Dirac operator on a large mass range, for a gauge configuration with $Q=-1$.

Follana is supported on the MICINN Ramón y Cajal program. and A. Vaquero was supported by MICINN through the FPU program.

\section{References}

[1] D. H. Adams, Phys. Rev. Lett. 104 (2010) 141602. [arXiv:0912.2850 [hep-lat]].

[2] V. Azcoiti, G. Di Carlo, E. Follana, A. Vaquero, in preparation.

[3] M. F. L. Golterman, Nucl. Phys. B273 (1986) 663.

[4] E. Follana Q. Mason, C.T.H. Davies, K. Hornbostel, G.P. Lepage, J. Shigemitsu, H. Trottier, K. Wong, Phys. Rev. D75 (2007) 054502. [hep-lat/0610092].

[5] E. Follana, A. Hart, C.T.H. Davies, Phys. Rev. Lett. 93 (2004) 241601. [hep-lat/0406010].

[6] E. Follana, A. Hart, C.T.H. Davies, Q. Mason, Phys. Rev. D72 (2005) 054501. [hep-lat/0507011].

[7] D. H. Adams, Phys. Lett. B699 (2011) 394-397. [arXiv:1008.2833 [hep-lat]].

[8] C. Hoelbling, Phys. Lett. B696 (2011) 422-425. [arXiv:1009.5362 [hep-lat]].

[9] P. de Forcrand, A. Kurkela, M. Panero, PoS LATTICE2010 (2010) 080. [arXiv:1102.1000 [hep-lat]]. 

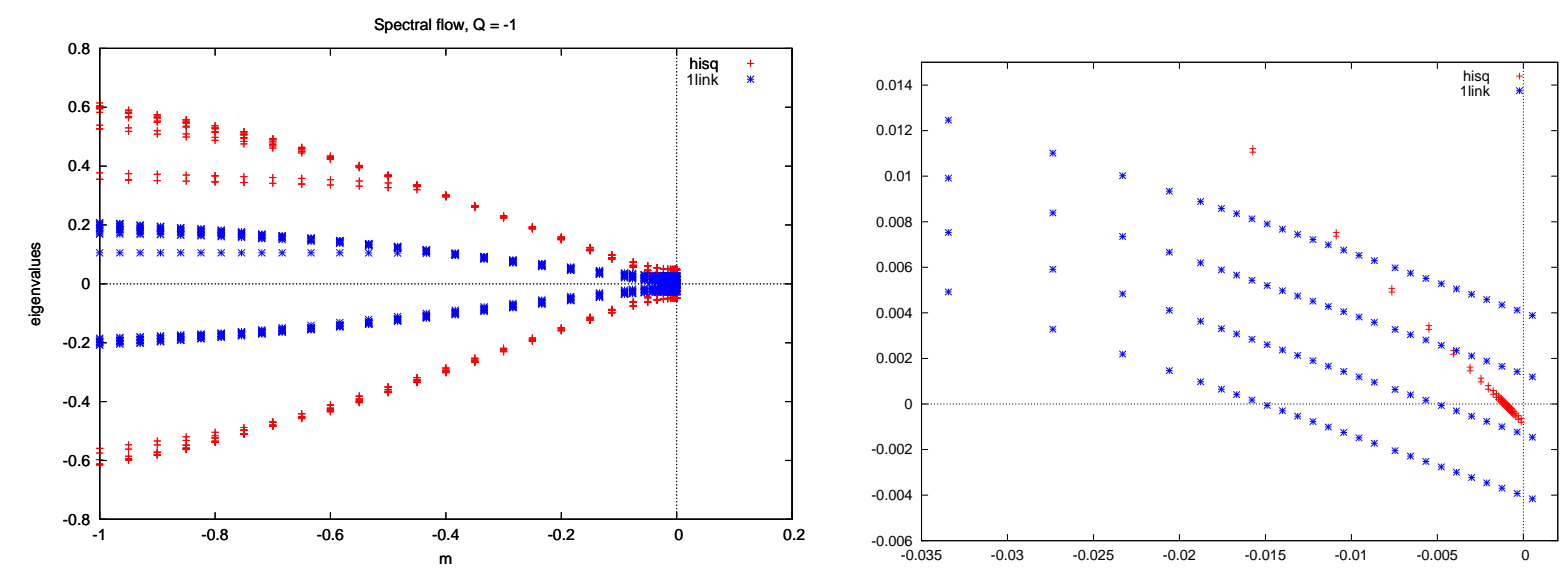

Figure 5: The spectral flow corresponding to the HISQ and the 1-link Dirac operators, on the same gauge field configuration.
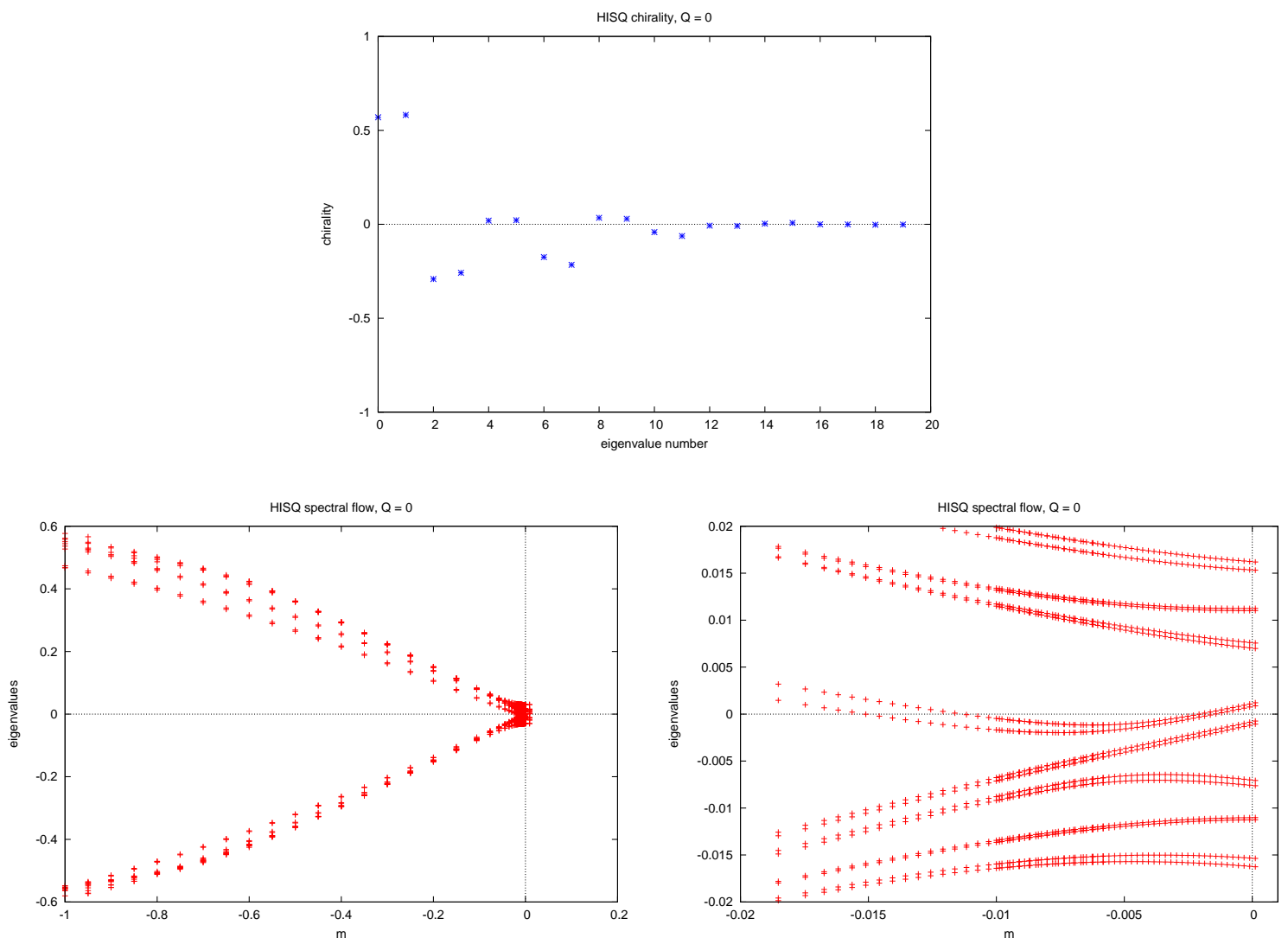

Figure 6: Chiralities and HISQ spectral flow for a configuration with an ambiguous topological charge, as determined by the chiralities. 